\title{
Optical materials based on molecular nano/microcrystals and ultrathin films
}

\author{
A PATRA, K RAJESH and T P RADHAKRISHNAN* \\ School of Chemistry, University of Hyderabad, Hyderabad 500 046, India
}

\begin{abstract}
Methodologies that we developed recently for the fabrication of molecular crystals with size variation in the nano to micro regime and polyelectrolyte templated mono and multilayer Langmuir-Blodgett films, are reviewed. The electronic absorption and strong fluorescence in the molecular nano/microcrystals are found to be size-dependent. Crystal structure and computational investigations provide a unified model to explain these observations. Role of polyelectrolyte templating in achieving stable and enhanced optical second harmonic generation response from LB films based on a hemicyanine amphiphile is highlighted.
\end{abstract}

Keywords. Molecular nano/microcrystals; aggregate fluorescence; Langmuir-Blodgett film; polyelectrolyte; second harmonic generation.

\section{Introduction}

Nanomaterials based on metals and semiconductors are the subject of extensive investigations because of their (i) accessibility through a wide range of fabrication routes including soft chemical methods, (ii) unique characteristics and attributes, and (iii) extensive application potential. There is growing interest in nanostructures constructed from molecular building blocks as well, because of the flexibility and versatility inherent in their assembly, novel size-dependent attributes they manifest, and the promise of emerging applications. Owing to the relatively weaker interactions between the building units, the hallmark of molecular materials, it is likely that some of the fundamental characteristics of molecular nanomaterials are different from those of metal and semiconductor nanostructures. For instance, the size-dependence of optical properties in molecular nanocrystals has been attributed to the variation in lattice softness and the consequent tuning of the strength of intermolecular interactions, rather than quantum confinement effect familiar in semiconductor nanocrystals (Wise 2000; Horn and Rieger 2001). Considerations such as these suggest that molecular nanostructures are highly promising from the perspectives of novel physical phenomena as well as technological applications.

Several methodologies involving 'build-up' as well as 'break-down' approaches have been developed for the synthesis of molecular nanomaterials (Ozin and Arsenault 2005). Nanocrystals have been fabricated through simple reprecipitation (Nakanishi and Oikawa 2003), vapour deposition (Cai et al 1998) and flash evaporation of solu-

*Author for correspondence (tprsc@uohyd.ernet.in) tions in supercritical fluid (Komai et al 1999) as well as sonochemical treatment (Bučar and MacGillivray 2007) and laser ablation methods (Masuhara and Asahi 2003). We have shown that a reprecipitation-digestion protocol can be employed to achieve size-tuning of the molecular nanocrystals (Patra et al 2006). Ultrathin films of molecules are commonly formed through self-assembly (Ulman 1996), layer-by-layer deposition (Laschewsky et al 1996; Decher 1997) and Langmuir-Blodgett (LB) film transfer (Petty 1996) on suitable substrates. A large number of variations on these basic themes have been developed over the years. We have shown that polyelectrolyte templating is a particularly versatile and efficient technique to overcome a number of fundamental limitations of the LB method, such as Langmuir film instability at the airwater interface (Sharma et al 2001) and molecular aggregation in the ultrathin films (Chandra et al 2003). Molecular nanostructures have been demonstrated to possess novel and unique properties and hence are suitable for several applications. Optical properties can be exploited in sensor and switching applications (Botzung-Appert et al 2004; Lim et al 2004), light emitting devices (Piok et al 2003) and photonics (Sanz et al 2001). Mono and multilayer molecular films are useful membrane models (Takano et al 1997; Zadmard et al 2004) and find applications in electronics and photonics (Martin et al 1993; Wijekoon et al 1996; Li et al 2001). Molecular scale devices of great current interest in areas such as spintronics, electronics and photonics (Kasai et al 1999) can be visualized as the logical extension of the molecular nanostructures.

We present here, an overview of two types of molecular nanostructures that we have been investigating in our laboratory in the recent past. We describe first, our studies of novel molecular nanocrystals: the methodology deve- 
loped for the fabrication of size-tuned nanocrystals, their optical properties and a new paradigm proposed to rationalize the size-dependence of these properties, based on spectroscopic, microscopic, crystallographic and computational investigations. Observation of strong blue fluorescence in the solution, colloid and solid states of a molecule will also be noted. Then we discuss our explorations of LB films of an amphiphilic molecule based on the hemicyanine chromophore, a prototype for quadratic nonlinear optical applications. Utility of a simple polyelectrolyte templating strategy for the fabrication of mono and multilayer films of this amphiphile showing stable and enhanced second harmonic generation (SHG) will be outlined.

\section{Fluorescent molecular materials: size effects in nano/micro regime}

Aggregation of fluorophore molecules often leads to effective suppression of the fluorescence; this is attributed to the so-called self-quenching effect. Molecular materials which show strong fluorescence in the aggregated state are therefore of considerable interest from the point of view of solid state optical devices. We have been studying a family of zwitterionic molecules based on the diaminodicyanoquinodimethane framework, which exhibits such phenomena. PMPDQ (scheme 1) is an example of a molecule which shows enhanced fluorescence in the solid state as well as in doped polymer films (Jayanty and Radhakrishnan 2004). The enhanced fluorescence in the solid state can be attributed to the arresting of molecular twist in the excited state and associated relaxation to a non-fluorescent structure, which most likely happens in the solution state. The phenomenon could be exploited for vapochromic switching of the fluorescence of doped polymer films.

The family of diaminodicyanoquinodimethanes including PMPDQ show green fluorescence whereas the corresponding anilino derivatives such as BCADQ (scheme 1)

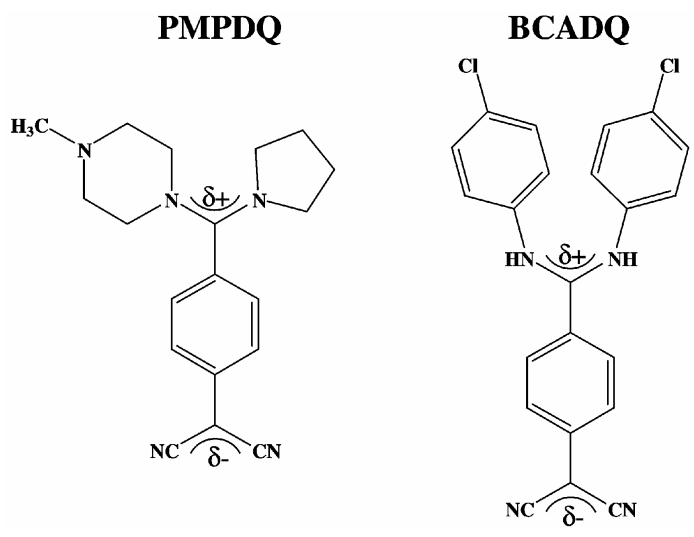

Scheme 1. possess extended conjugation into the aromatic groups on the diaminomethylene moiety and were found to exhibit red fluorescence (Patra et al 2006). More interestingly, BCADQ as well as similar halo derivatives were amenable to stable colloid formation through the reprecipitation route. We followed this up with a protocol involving digestion of the colloid at different temperatures (figure 1) which led to the formation of nano/microcrystals with sizes varying from $\sim 60 \mathrm{~nm}-2 \mu \mathrm{m}$ (table 1 ). TEM images of typical nanocrystalline plates of BCADQ are shown in figure 2. Electronic absorption and fluorescence emission spectra of BCADQ show strong changes between the solution and colloid/solid states as expected based on the impact of molecular aggregation and the resulting intermolecular interactions. The more significant observation is the profound changes in the absorption spectra and increase of fluorescence emission with the size of the nano/ microcrystals (figure 3); a detailed study of similar effects in a related molecule has been reported recently (Patra et al 2007b). The spectra of the colloids with crystal sizes of $\sim 2 \mu \mathrm{m}$ resemble closely those of the solid state material. The dramatic impact of crystal size is illustrated in figure 4 which shows increasing intensity of fluorescence from BCADQ colloids having identical content of the compound but increasing particle sizes. The spectroscopic studies revealed a number of interesting size-dependent optical properties in BCADQ nano/ microcrystals: (i) an unusually large Stokes shift in the colloidal state, especially with the smaller particle sizes, (ii) gradual broadening of the absorption spectrum with increasing particle size resulting from addition of new peaks and minor red shift of the individual peaks, and (iii) marginal blue shift of the emission peak, increasing emission intensity and increasing excited state lifetime with increasing particle size. Based on crystallographic characterization of BCADQ and semiempirical quantum chemical computations on the molecule and supramolecular clusters, we have developed a model that provides a rationale for all the above observations. The simplest model utilizes molecular dimers to assess the impact of intermolecular interactions. The salient features are: (i) the computed ground and excited states of the molecule and molecular dimers extracted from the solid state structure show good agreement with the absorption and emission energies of the molecule in solution and colloid/solid states, (ii) the nanocrystals being relatively soft compared to the larger ones, sustain only the strongest intermolecular interactions and (iii) with increasing size of the nano/microcrystals, not only do the individual intermolecular interactions become relatively stronger as is generally understood (Xiao et al 2003), but the impact of weaker intermolecular interactions get added on. The common emission is attributed to the monomer excited state which is populated by relaxation from the dimer excited state manifolds; the increase of intensity appears to be a 


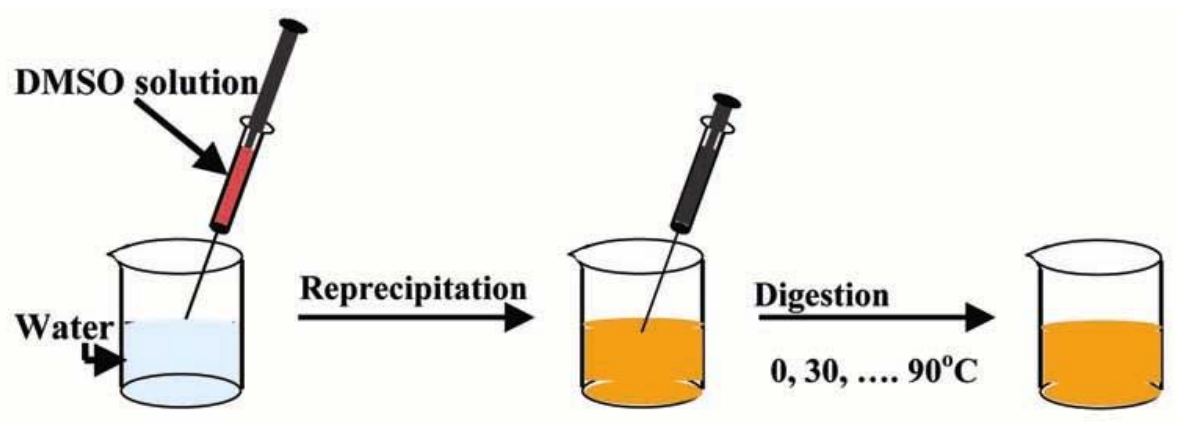

Figure 1. Reprecipitation/digestion method for the synthesis of molecular nano/ micro crystals with varying size.
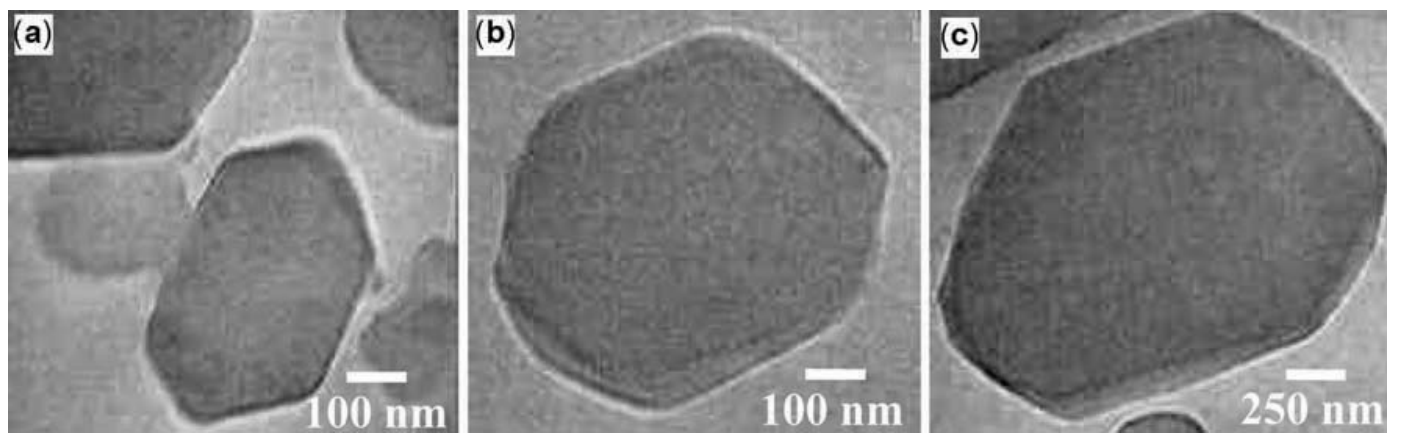

Figure 2. TEM images of BCADQ crystals obtained by digesting the colloid at different temperatures (a) 45 , (b) 60 and (c) $90^{\circ} \mathrm{C}$, revealing the size variation.

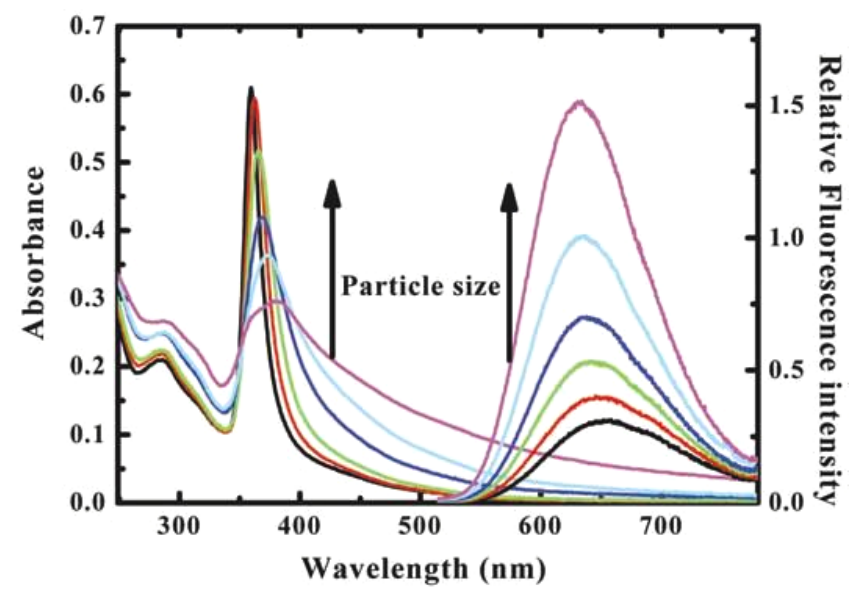

Figure 3. Progression of electronic absorption and emission spectra of BCADQ nano/microcrystals digested at $0,30,45,60$, 75 and $90^{\circ} \mathrm{C}$ resulting in increasing sizes.

Table 1. Range of sizes of the BCADQ crystals prepared by digestion at different temperatures; estimates are based on the smallest and largest dimensions of the flat faces of the dominant particles observed in the TEM images.

\begin{tabular}{lc}
\hline Colloid digestion temperature $\left({ }^{\circ} \mathrm{C}\right)$ & Size range $(\mathrm{nm})$ \\
\hline 0 & $60-100$ \\
30 & $100-250$ \\
45 & $200-500$ \\
60 & $300-600$ \\
75 & $500-1000$ \\
90 & $700-1800$ \\
\hline
\end{tabular}

consequence of the multiple channels opening up through the additional dimer states.

Following the investigations described above on green and red fluorescent diaminodicyanoquinodimethane solids, we have studied recently, a molecule which shows strong blue emission in the solution, colloid and solid states. Tris(4-cyanophenyl)amine (TCPA) was synthesized through a novel but intriguing procedure involving the direct reaction of 4-fluorocyanobenzene with potassium carbonate in DMSO (Patra et al 2007a). A complex mechanism was proposed to explain the covalent selfassembly of the three cyanophenyl units to form TCPA. Reprecipitation of TCPA by injection of acetonitrile solution into water gave highly monodisperse and well-defined nanocrystals (figure 5). Crystallographic characterization of TCPA showed supramolecular organizational motifs which can be visualized in terms of $\mathrm{H}$ and J-type interactions. Electronic absorption spectra showed the impact of molecular interactions in the colloid and solid states (figure $6)$. The solution spectrum shows a single absorption with $\lambda_{\max }$ at $338 \mathrm{~nm}$; the colloid and solid state spectra are broader with peaks at $378 \mathrm{~nm}$ and $365 \mathrm{~nm}$, respectively and a shoulder at $335-340 \mathrm{~nm}$. These observations could be rationalized using time-dependent density functional theory based computations on the molecule and its clusters extracted from the crystal structure. The emission spectra show strong enhancement on going from the solution to the solid state (figure 6). The $\lambda_{\max }$ for the solution is at 

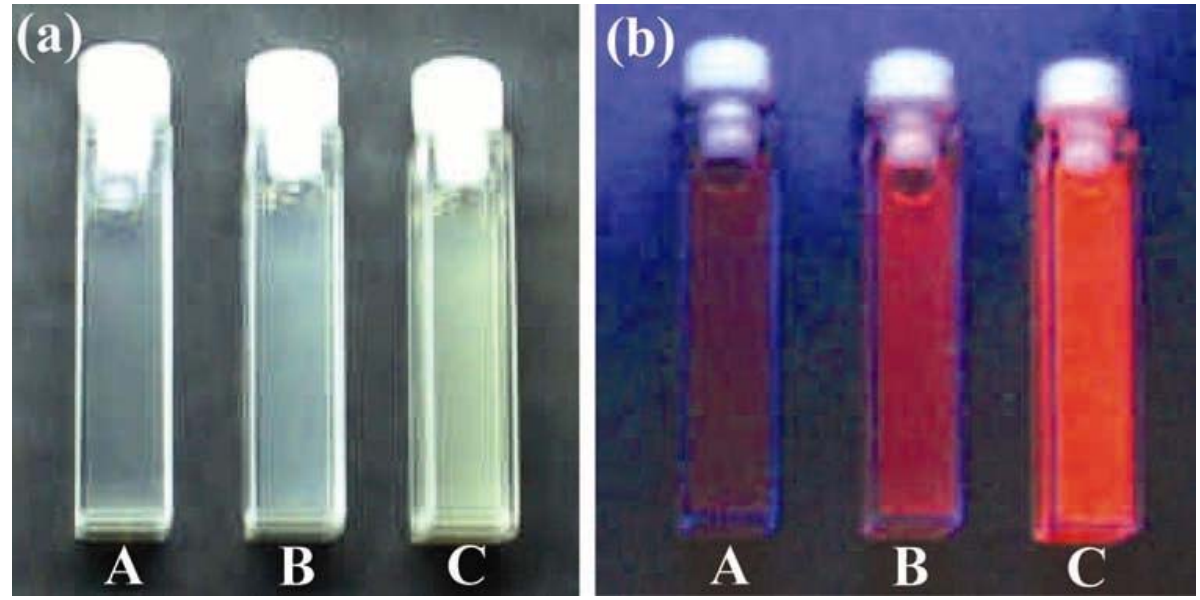

Figure 4. BCADQ colloids with same content of the compound, digested at 0 (A), 30 (B) and $90^{\circ} \mathrm{C}$ (C) under (a) normal and (b) UV (365 nm) light.

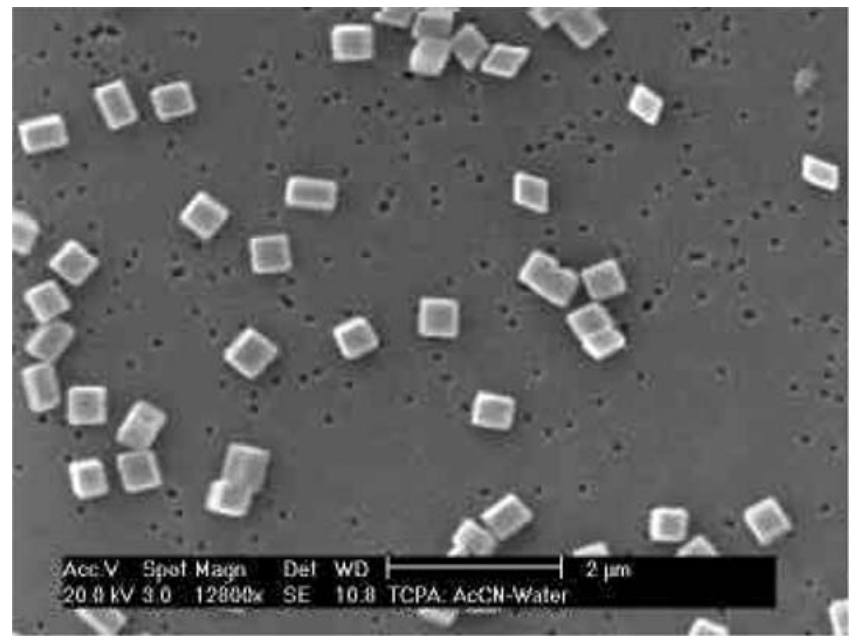

Figure 5. SEM image of TCPA crystals fabricated by reprecipitation.

$397 \mathrm{~nm}$; the colloid shows dual emission with peaks at $380 \mathrm{~nm}$ and $414 \mathrm{~nm}$ and the solid shows a broad emission with $\lambda_{\max }$ at $417 \mathrm{~nm}$ and a shoulder at $470 \mathrm{~nm}$. The computational studies suggest that the strong emission occurs from the electronic states generated by the different interaction motifs. The dual emission of the colloid may be attributed to excited states resulting from $\mathrm{H}$ and $\mathrm{J}$ type interactions. TCPA shows an unusually high melting temperature of $348^{\circ} \mathrm{C}$. The computations on oligomeric clusters extracted from the crystal lattice indicate that even though the intermolecular interactions are weak, they exhibit strong cooperative effects; this provides a basis to understand the high thermal stability of the material (Patra et al 2007a).

New members of the diaminodicyanoquinodimethane family of molecules which we have synthesized recently are susceptible to protonation-deprotonation equilibria and their solid state light emission are sensitive to the structural changes. Such molecules are of interest in sensor and switching applications. Molecular nano/microcrystals are amenable to embedding in polymer matrices facilitating device applications.

\section{Polyelectrolyte templated Langmuir-Blodgett films: second harmonic generation}

Langmuir-Blodgett transfer is one of the most elegant techniques for organized assembly of molecules into mono and multilayer ultrathin films. However, the methodology often encounters difficulties due to the instability of, or undesirable aggregation in the Langmuir films at the airwater interface and post-deposition molecular reorganizations due to the inherent metastability of the LB films. The latter problem, in particular, has limited the device applications of LB films. A commonly used strategy to address some of these difficulties is to fabricate LB films using an admixture of the desired amphiphile with molecules such as fatty acids which are known to form highly stable films (Girling et al 1987; Carpenter et al 1992). Even though the desired stability is generally achieved, this methodology leads to the dilution of the target amphiphile in the two-dimensional lattice and consequent reduction of the activity of interest; it can also lead to phase segregation. In this context, we have developed an alternate strategy of templating the amphiphiles using polyelectrolytes introduced from the aqueous subphase to impart stability to the Langmuir and LB films (figure 7). Amphiphiles with ionic headgroups are best served by this approach since efficient binding can be achieved through electrostatic complexation with polyelectrolytes such as poly(styrene sulfonate) (PSS), carboxymethyl cellulose (CMC), deoxyribonucleic acid (DNA) and poly(vinyl sulfate) (PVS). The utility of this approach was first de- 


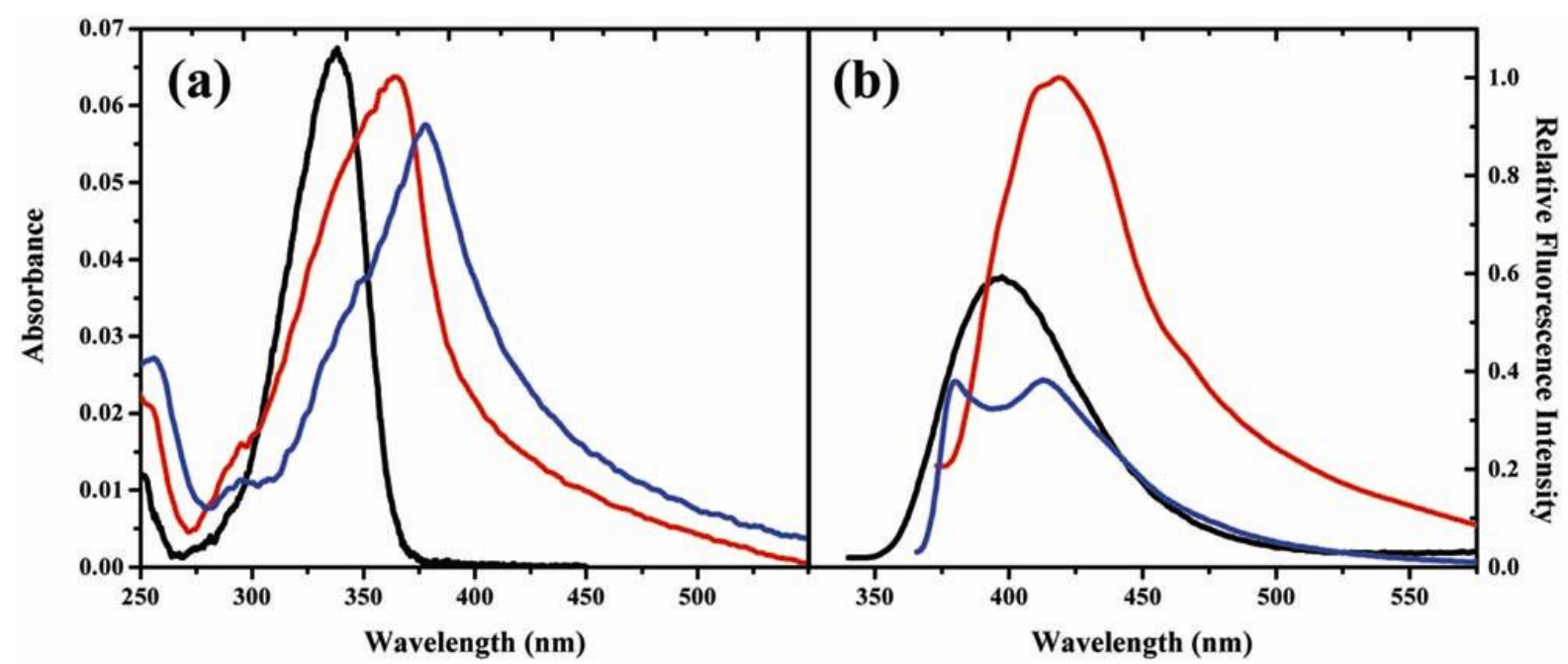

Figure 6. (a) Absorption and (b) fluorescence emission spectra of TCPA in solution (black), colloid (blue) and solid (red) states; the fluorescence spectra show the relative emission intensities.

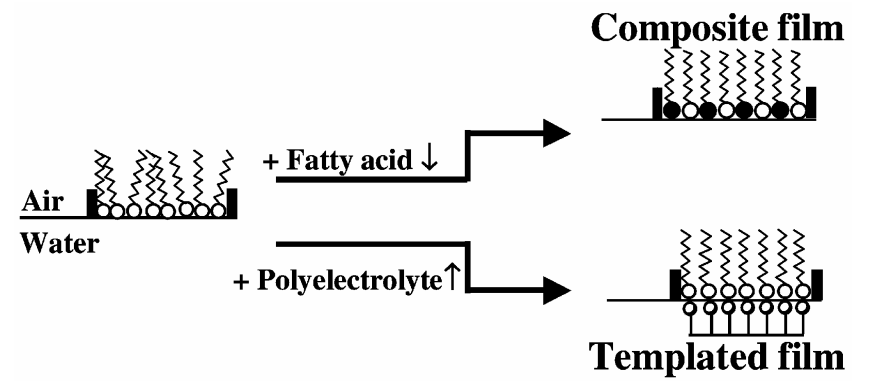

Figure 7. Schematic figure showing the methods to stabilize Langmuir films at the air-water interface using admixing of other amphiphiles to make composite films or polyelectrolyte templating.

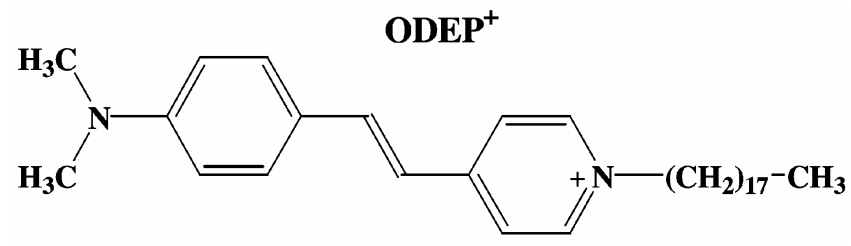

Scheme 2.

monstrated using a pyridinium based amphiphile; monolayers of this amphiphile at the air-water interface was found to collapse completely under compression, but to form a stable film when templated using PSS as well as some of its copolymers (Sharma et al 2001).

We have investigated in detail, the effect of polyelectrolyte templating on the formation of Langmuir film and stability of LB film of $\mathrm{ODEP}^{+}$(scheme 2), an amphiphile based on the prototypical hemicyanine push-pull system, and its SHG response. Polyelectrolytes introduced in the subphase have considerable impact on the morphology of the $\mathrm{ODEP}^{+}$monolayer at the air-water interface as revealed by Brewster angle microscopy (BAM). Figure 8 shows the formation of smooth films in presence of CMC. SHG of monolayer LB film of $\mathrm{ODEP}^{+} / \mathrm{Br}^{-}$is considerably reduced due to molecular aggregation effects which creates blue-shifted absorption near $360 \mathrm{~nm}$ in addition to the absorption due to monomeric $\mathrm{ODEP}^{+}$at 453 $\mathrm{nm}$ (Chandra et al 2004) (figure 9a). LB films formed in presence of PSS on the other hand, show no aggregation (figure 9b); the small absorption near $360 \mathrm{~nm}$ in this case is due to the polyelectrolyte. Consequently, the response is approximately four times stronger in the templated LB film (Chandra et al 2003). AFM images suggest that micron sized domains formed by $\mathrm{ODEP}^{+}$are broken up into network structures as a result of the complexation with polyelectrolytes (Chandra et al 2005). More interestingly, continued laser irradiation is found to enhance the aggregation of the hemicyanine head group in the case of $\mathrm{ODEP}^{+} /$ $\mathrm{Br}^{-}$as reflected in the change in absorption spectrum whereas such instabilities are effectively arrested in $\mathrm{ODEP}^{+} / \mathrm{PSS}^{n-}$ (figures 9a, b). The impact on SHG is seen as degradation of the response to about $10 \%$ of the original, following repeated laser irradiations in the case of the former, whereas it does not reduce below $90 \%$ in the case of the latter (Chandra et al 2005).

Polyelectrolyte templating exerts strong influence on the formation of multilayer LB films as well. The transfer is nearly $Z$-type in the case of $\mathrm{ODEP}^{+} / \mathrm{Br}^{-}$as well as $\mathrm{ODEP}^{+} / \mathrm{CMC}^{n-}$. However, it is nearly $Y$-type when polyelectrolytes such as DNA or PSS are employed. The linear growth of the electronic absorption intensity supports the formation of homogeneous multilayer films of $\mathrm{ODEP}^{+} /$ $\mathrm{Br}^{-}$and $\mathrm{ODEP}^{+} / \mathrm{PSS}^{n-}$ (figures 9c, d); the spectra for different multilayer films in the two cases show $\lambda_{\max }$ at 

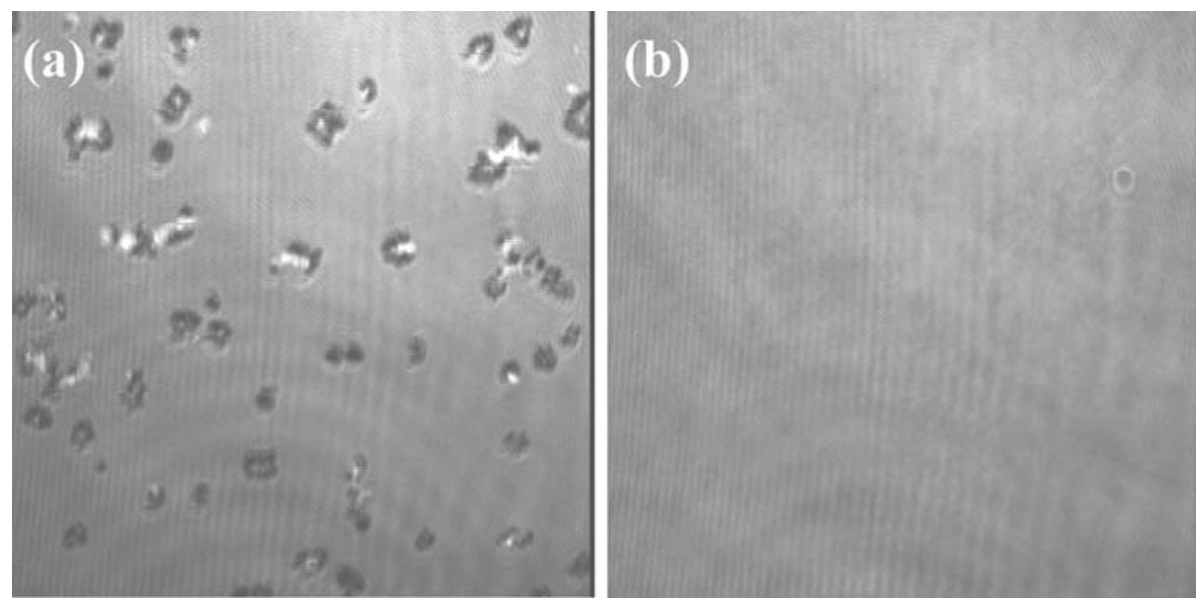

Figure 8. BAM images of Langmuir films of (a) $\mathrm{ODEP}^{+} / \mathrm{Br}^{-}$and (b) $\mathrm{ODEP}^{+} / \mathrm{CMC}^{n-}$ at the air-water interface subjected to $20 \mathrm{mN} / \mathrm{m}$ pressure.
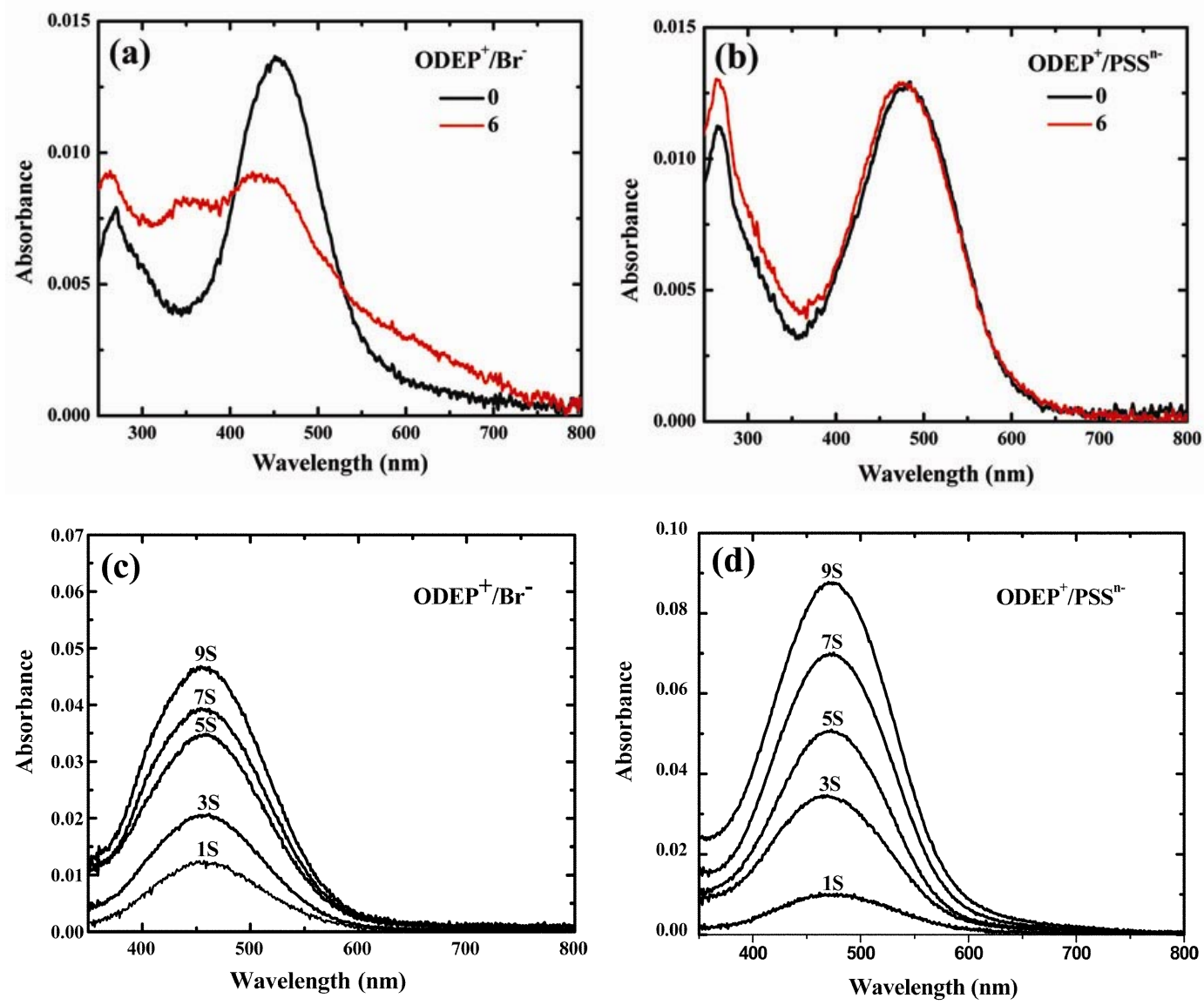

Figure 9. Electronic absorption spectra of monolayer $\mathrm{LB}$ films of (a) $\mathrm{ODEP}^{+} / \mathrm{Br}^{-}$and (b) $\mathrm{ODEP}^{+} / \mathrm{PSS}^{n-}$ before laser irradiation and after six irradiations, and multilayer LB films of (c) $\mathrm{ODEP}^{+} / \mathrm{Br}^{-}$and (d) $\mathrm{ODEP}^{+} / \mathrm{PSS}^{n-}$ (the number of strokes are indicated).

$\sim 458 \mathrm{~nm}$ and $\sim 472 \mathrm{~nm}$, respectively. Detailed studies including analysis of transfer ratios, electronic absorption spectroscopy, atomic force microscope characterization and nonlinear optical measurements show that CMC is the optimal choice of polyelectrolyte to effect efficient multilayer transfer of $\mathrm{ODEP}^{+}$leading to LB films that show the expected quadratic growth of SHG efficiency with material transfer (Rajesh et al 2007). It appears that oriented assembly of the nonlinear optical chromophores is achieved through suitable adjustment of the hydropho- 
bic/hydrophilic regions of the sandwiching polyelectrolyte layers. Optical and nonlinear optical responses of the multilayer films of $\mathrm{ODEP}^{+} / \mathrm{CMC}^{n-}$ show very good stability when subjected to extended laser irradiation; this may be attributed to the interlayer gluing effected by the electrostatic interactions with the polyelectrolyte layers.

\section{Conclusions}

Two approaches developed in our laboratory for the tailored assembly of molecules are described. The first one involves the colloidal route to nano/microcrystals of molecules that exhibit strong fluorescence in the aggregated state. Significant impact of crystal size on the optical properties and a new model proposed to explain these observations are described. The second topic relates to Langmuir-Blodgett films based on molecules of interest in quadratic nonlinear optical applications. Contrary to the case of the nano/microcrystals, molecular aggregation has a deleterious effect in this case; the second harmonic generation capability of these ultrathin films are diminished due to aggregation. The efficient solution to this problem based on polyelectrolyte templating is described. Current efforts in our laboratory are focused on the exploitation of these molecular nanostructures for potential applications in sensors and photonics.

\section{Acknowledgements}

Financial support from DST (NSTI program) is gratefully acknowledged. We thank Dr B Sreedhar for help with the microscopy studies and Prof. J Kawamata for the second harmonic generation measurements on LB films. (AP) thanks UGC and (KR) thanks CSIR for senior research fellowships. Visit of (KR) to Yamaguchi University to carry out some of the SHG experiments was supported by an IndoJapan collaborative project funded by DST and JSPS.

\section{References}

Botzung-Appert E, Monnier V, Duong T H, Pansu R and Ibanez A 2004 Chem. Mater. 161609

Bučar D and MacGillivray L R 2007 J. Am. Chem. Soc. 12932

Cai C et al 1998 J. Am. Chem. Soc. 1208563

Carpenter M A, Willand C S, Penner T L, Williams D J and Mukamel S J 1992 J. Phys. Chem. 962801

Chandra M S, Ogata Y, Kawamata J and Radhakrishnan T P 2003 Langmuir 1910124
Chandra M S, Ogata Y, Kawamata J and Radhakrishnan T P 2004 J. Nonlinear Opt. Phys. Mater. 13347

Chandra M S, Krishna M G, Mimata H, Kawamata J, Nakamura T and Radhakrishnan T P 2005 Adv. Mater. 171937

Decher G 1997 Science 2771232

Girling I R et al 1987 J. Opt. Soc. Am. B 4950

Horn D and Rieger J 2001 Angew. Chem. Int. Ed. 404330

Jayanty S and Radhakrishnan T P 2004 Chem. Eur. J. 10791

Kasai H, Oikawa H and Nakanishi H 1999 Organic mesoscopic chemistry (eds) H Masuhara and F C de Schryver (Oxford: Blackwell Science) p. 145

Komai Y et al 1999 Jpn J. Appl. Phys. 38 L81

Laschewsky A, Mayer B, Wischerhoff E, Arys X, Bertrand P, Delcorte A and Jona A 1996 Thin Solid Films 334284

Li F, Jin L, Huang C, Zheng J, Guo J, Zhao X and Liu T 2001 Chem. Mater. 13192

Lim S, An B, Jung S D, Chung M and Park S Y 2004 Angew. Chem. Int. Ed. 436346

Martin A S, Sambles J R and Ashwell G J 1993 Phys. Rev. Lett. 70218

Masuhara H and Asahi T 2003 Single organic nanoparticles (eds) H Masuhara et al (Berlin: Springer) p. 32

Nakanishi H and Oikawa H 2003 Single organic nanoparticles (eds) H Masuhara et al (Berlin: Springer) p. 17

Ozin G A and Arsenault A C 2005 Nanochemistry: A chemical approach to nanomaterials (Cambridge: Royal Society of Chemistry)

Patra A, Hebalkar N, Sreedhar B, Sarkar M, Samanta A and Radhakrishnan T P 2006 Small 2650

Patra A, Anthony S P and Radhakrishnan T P 2007a $A d v$. Funct. Mater. 172077

Patra A, Hebalkar N, Sreedhar B and Radhakrishnan T P 2007b J. Phys. Chem. C 11116184

Petty M C 1996 Langmuir-Blodgett films (Cambridge: University Press)

Piok T et al 2003 Adv. Mater. 15800

Rajesh K, Chandra M S, Hirakawa S, Kawamata J and Radhakrishnan T P 2007 Langmuir 238560

Sanz N, Ibanez A, Morel Y and Baldeck P L 2001 Appl. Phys. Lett. 782569

Sharma S, Chandra M S and Radhakrishnan T P 2001 Langmuir 178118

Takano E, Ishida Y, Iwahashi M, Araki T and Iriyama K 1997 Langmuir 135782

Ulman A 1996 Chem. Rev. 961553

Wijekoon W M K P, Wijaya S K, Bhawalkar J D, Prasad P N, Penner T L, Armstrong N J, Ezenyilimba M C and Williams D J 1996 J. Am. Chem. Soc. 1184480

Wise F W 2000 Acc. Chem. Res. 33773

Xiao D, Xi L, Yang W, Fu H, Shuai Z, Fang Y and Yao J 2003 J. Am. Chem. Soc. 1256740

Zadmard R, Arendt M and Schrader T 2004 J. Am. Chem. Soc. 1267752 